\title{
Converging power functions as a description of the size-weight illusion: A control experiment
}

\author{
STANLEY J. RULE and DWIGHT W. CURTIS \\ University of Alberta, Edmonton, Alberta, Canada T6G $2 E 9$
}

\begin{abstract}
Stevens and Rubin (1970) and Cross and Rotkin (1975) found that the exponent for magnitude estimation of heaviness seemed to increase with increasing volume of the object lifted such that the data could be described by a set of converging power functions. For their set of objects, weight was partially confounded with volume. If the exponent for heaviness is not constant over a large range of weight, their data could have resulted from changes in the exponent with increasing weight rather than increasing volume.. To control for this possibility, 10 subjects gave magnitude estimation of heaviness of objects with the same weight as in the Stevens and Rubin's study, but the objects were hidden from the subjects' view. A single power function with an exponent of .92 provided a good fit to the data, indicating that the original results were not an artifact of the unbalanced stimulus combinations.
\end{abstract}

The size-weight illusion refers to a phenomenon in which objects of constant weight appear lighter when volume is increased. From an investigation of the illusion, Stevens and Rubin (1970) found that the relation between magnitude estimation of heaviness and physical weight could be described by the following power function in which the coefficient and exponent vary with volume:

$$
\mathbf{Y}=\mathbf{a}_{\mathbf{j}} \mathbf{X}^{\mathrm{n}_{\mathbf{j}}}
$$

where $\mathrm{Y}$ denotes a magnitude estimate of the heaviness of an object with physical weight $X$. The coefficient $a_{j}$ and exponent $n_{j}$ are specific to objects with the $\mathrm{jth}$ volume. The exponent tended to increase, and the coefficient tended to decrease with increasing volume such that the set of power functions implied by Equation 1 appeared to converge to a point beyond the range of stimuli. This result has been replicated by Cross and Rotkin (1975), who also found that the relation between the coefficient $a_{j}$ and volume could be approximated closely by a power function with a negative exponent.

With respect to the ranges of weight and volume studied, the Stevens and Rubin and the Cross and Rotkin studies are the most extensive investigations of the size-weight illusion that have been published. It is important to use a large range of stimulus values because it provides an opportunity to examine trends in data that might be too subtle to detect otherwise. However, these investigators' use of a large stimulus range created a difficulty in the construction of their stimuli. The stimuli in the two studies were either polyethylene bottles or plastic cylinders that were filled with lead. Because of

This research was supported by Grants A0151 and A9582 from the National Research Council of Canada. physical limitations, all weight measures could not be combined with all volumes. The smaller containers could not contain as much lead as was required by the upper extreme of the weight range, while the weight of the larger containers was greater when empty than was required by the lower values of the range. Consequently, the weight of their objects tended to increase with volume. Because of this, their exponents increased not only with volume, but with the weight of the series, as well. It is conceivable, therefore, that the exponent for perceived heaviness may not remain constant over very large ranges of weight, regardless of the influence of volume. Findings by Stevens and Rubin and by Cross and Rotkin could have been an artifact of their experimental design if the heaviness exponent increases with increasing weight of the series due to, say, using different muscles.

Because the results could have been an artifact of an unbalanced design, it is important to investigate this possibility before a set of converging power functions can be accepted as a mathematical description of the size-weight illusion. The present study represents the necessary control experiment. It was essentially a replication of Stevens and Rubin's study, except that the objects lifted were hidden from the subjects. By partitioning the data into sets corresponding to each uniform volume series in Stevens and Rubin's original study, it was possible to determine what effect the weight of a series had on the value of the exponent in the absence of volume cues.

\section{METHOD}

A set of nine weights was constructed which had the same physical weights as the objects used by Stevens and Rubin (1970). Their weight ranged from 25 to $6,400 \mathrm{~g}$ in equal logarithmic 
steps. The apparatus used for lifting the weights was similar to that described by Curtis, Attneave, and Harrington (1968). Nylon fishline was passed through a hole in the top of a table. A wooden drawer knob, $3.2 \mathrm{~cm}$ in diam, was attached to the upper end of the line, and a hook was attached to the lower end on which weights could be suspended. The subject lifted the weight by raising the wooden knob. Except for the knob, the subject had no information about the volume of the object lifted. The volume of the knob was $9 \mathrm{ml}$.

Ten students, enrolled in an introductory psychology course, served as subjects. The subjects gave magnitude estimates of two series of 40 presentations of weights. The subjects were free to choose their own modulus for making their estimates. The two series of presentations differed only with respect to random order of presentation. Each of nine weights was presented in the series with the same frequency with which its physical weight appeared in Stevens and Rubin's study. The frequency of presentation of each weight is indicated by the filled entries in Table 1.

\section{RESULTS AND DISCUSSION}

Magnitude judgments for each series of 40 presentations were partitioned into seven sets of data. Each set consisted of data corresponding to one of Stevens and Rubin's uniform volume series. The set to which a response to a stimulus was assigned was determined randomly with the restriction that each set contain data for the same physical weight as were in Stevens and Rubin's uniform volume series. Geometric mean responses for each set are presented in Table 1. Also presented in Table 1 are estimates of the coefficient and exponent of a power function fitted separately to data within each set. The functions were fitted by the method of least squares applied to logarithmically transformed stimulus and response measures. The coefficients appear to exhibit little or no trend over the seven sets, while the trend in the exponents is an initial increase followed by a decrease in value.

A plot of geometric mean response to each weight

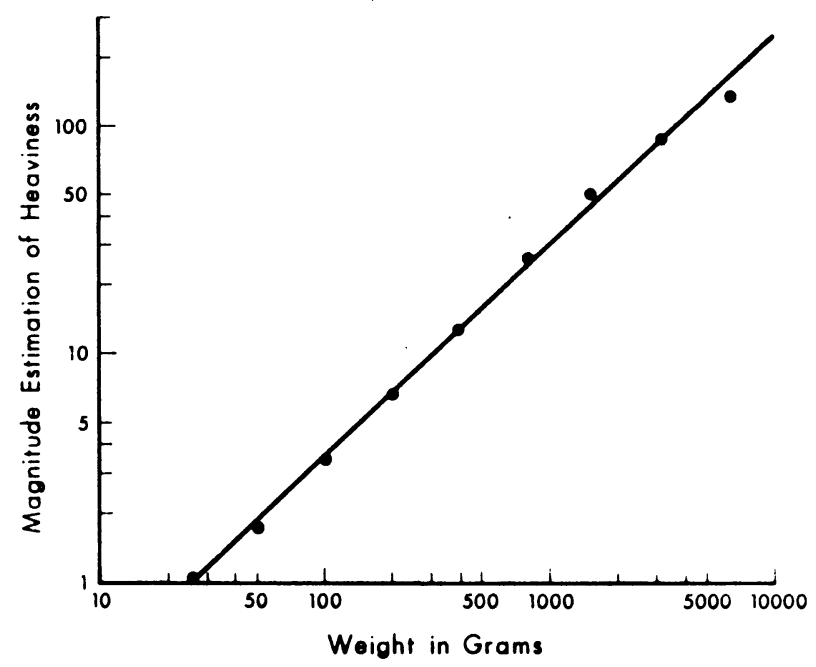

Figure 1. Magnitude estimation of heaviness plotted as a function of weight. The slope of the line through the data is .92 .

pooled over presentations against physical weight is presented on logarithmic coordinates in Figure 1. The line through the data was fitted by a weighted least-squares solution in which each point was weighted by the number of observations on which it was based. The slope (exponent) of the line is .92 . A hypothesis that the exponent increases with the weight of the series would require the plot to be concaved upward. Clearly, it is not. Instead, there is a slight tendency for the plot to flatten at the ends. This is consistent with the smaller estimates of the exponents in Table 1 for the first and last two sets of data. Overall, however, a power function appears to provide a good approximation to the data from the entire range of weights used by Stevens and Rubin. Also, extrapolation from the trend in Stevens and Rubin's exponents to zero volume provides an estimate of the weight exponent that is close

Table 1

Geometric Mean Magnitude Estimation for Heaviness and Parameters of Power Functions for Seven Data Sets Corresponding to Each of Stevens and Rubin's Uniform Volume Series

\begin{tabular}{|c|c|c|c|c|c|c|c|}
\hline \multirow[b]{2}{*}{ Grams } & \multicolumn{7}{|c|}{ Data Set } \\
\hline & 1 & 2 & 3 & 4 & 5 & 6 & 7 \\
\hline & \multicolumn{7}{|c|}{ Geometric Mean } \\
\hline 6400 & & & & & & 133.09 & 137.84 \\
\hline 3200 & & & & & 91.47 & 87.41 & 88.80 \\
\hline 1600 & & & & 51.71 & 46.13 & 51.62 & 52.11 \\
\hline 800 & & 27.74 & 25.42 & 25.58 & 24.21 & 26.75 & 27.94 \\
\hline 400 & 12.49 & 13.15 & 12.57 & 11.97 & 13.94 & 14.13 & 12.33 \\
\hline 200 & 7.29 & 6.74 & 5.82 & 6.42 & 6.96 & 6.71 & 7.81 \\
\hline 100 & 3.57 & 3.38 & 3.67 & 3.92 & 3.42 & & \\
\hline 50 & 1.92 & 1.55 & 1.57 & 1.98 & & & \\
\hline \multirow[t]{2}{*}{25} & 1.09 & 1.05 & & & & & \\
\hline & \multicolumn{7}{|c|}{ Parameters } \\
\hline Coefficient & .06 & .04 & .04 & .05 & .05 & .08 & .08 \\
\hline Exponent & .90 & .97 & .98 & .93 & .93 & .87 & .86 \\
\hline
\end{tabular}


to .92 , the exponent from the present analysis. It must be concluded, therefore, that Stevens and Rubin's results, and Cross and Rotkin's findings as well, were not an artifact of using different distributions of weights.

\section{REFERENCES}

CRoss, D. V.. \& Rotkin, L. The relation between size and apparent heaviness. Perception \& Psychophysics, 1975. 18. 79.87

Curtis. D. W.. Attneave, F., \& Harrington, T. L. A test of a two-stage model of magnitude judgment. Perception \& Psychophysics. 1968, 3, 25-31.

Stevens, J. C., \& Rubin, L. L. Psychophysical scales of apparent heaviness and the size-weight illusion. Perception \& Psychophysics, 1970, 8, 225-230.

(Received for publication March 19, 1976.) 